\title{
Zertifizierte Gefäßzentren der DRG
}

Die Deutsche Röntgengesellschaft führt in Abstimmung mit der Deutschen Gesellschaft für Gefäßchirurgie (DGG) und der Deutschen Gesellschaft für Angiologie (DGA) Zertifizierungen von Gefäßzentren durch.

Berlin, Januar 2014. Die Deutsche Röntgengesellschaft (DRG) unterstützt gemeinsam mit der Deutschen Gesellschaft für Angiologie (DGA) und der Deutschen Gesellschaft für Gefäßchirurgie (DGG) die qualitätsgesicherte Behandlung von Gefäßerkrankungen in Deutschland mit einer durch alle drei Fachgesellschaften anerkannten Zertifizierung von interdisziplinären Gefäßzentren. Die Vorteile der hierbei geforderten interdisziplinären Entscheidungsfindung liegen klar auf der Hand: Therapiekonzepte werden in interdisziplinären Fallkonferenzen festgelegt. Für den Patienten bedeutet dies eine Rundumversorgung durch Gefäßchirurgie, Angiologie und Radiologie. Der Fokus liegt dabei auf dem Erreichen eines optimalen Behandlungsergebnisses und ist damit ganz auf das Patientenwohl abgestimmt.

\section{Warum Gefäßzentren?}

Die Diagnose und Therapie von Gefäßerkrankungen haben sich in den letzten Jahren dynamisch weiterentwickelt. Neben neuen aussagekräftigen bildgebenden Verfahren wurden zahlreiche innovative interventionelle Behandlungsverfahren entwickelt. Ferner zeigen die demographischen und epidemiologischen Daten, dass die Diagnose und Therapie von Gefäßerkrankungen in den nächsten Jahren und Jahrzehnten eine wachsende Bedeutung gewinnen werden, wobei zunehmend polymorbide Patienten zu behandeln sind.

Aufgrund dieser Umstände treten zunehmend interdisziplinäre Behandlungsansätze in den Vordergrund. Dies hat in zahlreichen Einrichtungen dazu geführt, dass fachübergreifende Diagnose- und Therapiekonzepte in ambulanten und stationären Einrichtungen zum Tragen kommen. Dabei kommt der diagnostischen und interventionellen Radiologie eine zentrale Bedeutung zu, die sie am besten in Kooperation mit der Gefäßchirurgie sowie Angiologie und den übrigen assoziierten Fachgebieten ausfüllen kann. Die Bildung eines Gefäßzentrums ist die logische Konsequenz. Dabei sollen auch die Verbindungen zwischen der ambulanten und stationären Versorgung gestärkt werden.

\section{Warum Zertifizierung von Gefäß-} zentren?

Der Zertifizierungsprozess zum anerkannten Gefäßzentrum soll u.a. dazu beitragen, die Abläufe noch klarer zu strukturieren, Verantwortlichkeiten eindeutig zuzuweisen, Strukturveränderungen anstoßen, die Interdisziplinarität zu stärken und letztendlich so einen entscheidenden Anteil zu Qualitätsverbesserung und -sicherung beitragen. Ziel der DRG und der beteiligten Gesellschaften ist es, den Patienten dabei noch stärker in den

Fokus zu rücken. Von den Beteiligten wird erwartet, dass sie einen ganzheitlichen Ansatz zur Lösung des individuellen Gefäßproblems des einzelnen Patienten erarbeiten.

Im zertifizierten Gefäßzentrum soll eine sachgerechte, serviceorientierte Arbeitsteilung ermöglicht werden, um so den zukünftigen Anforderungen noch flexibler und effizienter gerecht zu werden.

\section{Die Ziele der Zertifizierung von Gefäßzentren}

Die Ziele der Abstimmung der drei Fachgesellschaften bei der Zertifizierung von Gefäßzentren lassen sich wie folgt zusammenfassen.

- Die Organisationsstruktur wird patientengerechter.

- Es wird eine stärkere „Kundenorientierung" und Objektivierung erzielt.

- Das Profil, die Kompetenz und die Wettbewerbsposition der Kliniken werden gestärkt.

- Die Ansprechbarkeit für andere Kliniken und Hausärzte wird verbessert, die Antwortzeiten lassen sich deutlich reduzieren.

- Sämtliche Abläufe werden auf den Gefäßpatienten zentriert, woraus sich eine sachgerechte, qualitativ verbesserte, innovative und letztendlich kostengünstigere Patientenversorgung ergibt. Die zu erfüllenden Voraussetzungen zur Zertifizierung sowie den genauen Ablauf finden Sie auf der Homepage der DRG: http://www.drg.de/de-DE/1285/zertifizierte-gefaesszentren-der-drg Bei Fragen zur Zertifizierung von Gefäßzentren durch die DRG, DGA und DGG steht Ihnen Frau Dr. Rebecca Epsch (Tel.: 030916070-21, epsch@drg.de) gerne zur Verfügung. 